\title{
Radiotherapy, tumor mutational burden, and immune checkpoint inhibitors: time to do the math
}

\author{
Frank A. Giordano' - Marlon R. Veldwijk' - Carsten Herskind ${ }^{1}$ · Frederik Wenz ${ }^{1}$
}

Received: 25 June 2018 / Accepted: 12 July 2018 / Published online: 20 July 2018

○) Springer-Verlag GmbH Germany, part of Springer Nature 2018

Immune checkpoint inhibitors (ICI) are revolutionizing lung and skin cancer therapy [1-7], and there is accumulating evidence for a further significant and highly relevant boost of progression-free and overall survival once these substances are combined with radiotherapy (RT) $[8,9]$.

The tumor mutational burden (TMB) and the corresponding increase in neoantigen formation subsequent to exon mutations correlate with response after treatment with ICI $[10,11]$. Large studies of mutational patterns have shown the highest TMB in melanoma, lung, and bladder cancers, although considerable variation is present within individual tumor entities $[12,13]$. In this regard, the CheckMate 227 trial recently established a TMB of $\geq 10$ mutations per $10^{6}$ bases $(=1$ megabase; $\mathrm{Mb})$ as a robust and independent biomarker of response [14]. These data recently led radiation oncologists to speculate whether the improved efficacy of ICI plus RT may be caused by radiation-induced TMB.

We propose to take a closer look at this hypothesis by performing a simple calculation: Assume that 40 DNA double-strand breaks (DSBs), 1000 DNA single-strand breaks (SSBs), and 3000 base lesions are induced per Gy in the genome [15]. SSBs and base lesions arise in their thousands every day as a result of physiological processes in all cells and are repaired with extremely high efficiency. Even the vast majority of DSBs are repaired so that less than one DSB remains (per cell) after a daily fraction size of $2 \mathrm{~Gy}$. However, induced mutations adding to the TMB result from incorrectly repaired rather than unrepaired lesions. Approximately $15 \%$ of DSBs are repaired by error-free homologous recombination (HR) and the rest by non-homologous end joining (NHEJ) which may introduce small deletions or insertions ("indels") if simple end joining is not possible.

Frank A. Giordano, MD

frank.giordano@umm.de

1 Department of Radiation Oncology, University Medical Center Mannheim, Medical Faculty Mannheim, University of Heidelberg, Theodor-Kutzer-Ufer 1-3, 68167 Mannheim, Germany
This may result if end resection occurs or spurious homologies are present. Furthermore, misrepair may join unrelated DSB ends and fuse different genes or chromosomes. Assuming a probability of $10 \%$ for indels and a ratio of 8:1 base substitutions [16], a typical RT scheme of $60 \mathrm{~Gy}$ in 30 fractions of $2 \mathrm{~Gy}$ might produce $8 \times 30=240$ indels and approximately 2000 base substitutions. In the worst case, where most DSBs repaired by NHEJ would lead to indels, $50 \times 30=1500$ indels and thus 12,000 base substitutions might be produced. The range of $240-1500$ indels is consistent with the range of 135-943 indels and 6-321 rearrangement break points found per genome in 12 radiationinduced tumors [16]. Considering that the genome contains $6.6 \times 10^{9}$ bases, a standard RT scheme is unlikely to introduce more than 1 mutation per $\mathrm{Mb}$.

Thus, in standard-fractionated RT, it is unlikely that radiation-induced DNA modifications sufficiently raise the MTB above the critical limit of 10 per Mb.

In the case of RT using very large doses per fraction (such as in Stereotactic Body Radiotherapy [SBRT], Stereotactic Radiosurgery [SRS], brachytherapy or Intraoperative Radiotherapy [IORT]), error-free DNA damage repair becomes increasingly saturated and the rate of misrepaired DNA alterations may be considerably higher [17]. In line with this, clinical evidence arises from studies on ICI combined with SRS or SBRT that showed impressive responses and improved overall survival rates [18-23]. However, until quantitative yields of misrepair after high single doses become available, it is not possible to assess whether the MTB is high enough to explain these results. An alternative interpretation of the data is the function of RT as an immunological adjuvant creating an anti-tumor vaccine based on existing tumor antigens via necrotic or immunogenic cell death associated with an inflammatory response [24-29].

RT increases TMB, but the math does not support the hypothesis that conventionally fractionated RT increases the MTB enough to induce exploitable tumor antigens. The increase in TMB after a course of conventionally fractionated RT is one magnitude below the proposed threshold of 10 mutations per $\mathrm{Mb}$ for the induction of immune ef- 
fects in combination with ICI, indicating that other factors may be relevant for RT-induced immune stimulation [30]. It is plausible that the picture is different after high single doses of RT, where alternative mechanisms may explain the interaction of RT and ICI.

Conflict of interest F.A. Giordano, M.R. Veldwijk, C. Herskind, and F. Wenz declare that they have no competing interests.

\section{References}

1. Hodi FS, O'Day SJ, McDermott DF, Weber RW, Sosman JA, Haanen JB, Gonzalez R, Robert C, Schadendorf D, Hassel JC, Akerley W, van den Eertwegh AJ, Lutzky J, Lorigan P, Vaubel JM, Linette GP, Hogg D, Ottensmeier CH, Lebbe C, Peschel C, Quirt I, Clark JI, Wolchok JD, Weber JS, Tian J, Yellin MJ, Nichol GM, Hoos A, Urba WJ (2010) Improved survival with ipilimumab in patients with metastatic melanoma. N Engl J Med 363:711-723

2. Larkin J, Chiarion-Sileni V, Gonzalez R, Grob JJ, Cowey CL, Lao CD, Schadendorf D, Dummer R, Smylie M, Rutkowski P, Ferrucci PF, Hill A, Wagstaff J, Carlino MS, Haanen JB, Maio M, Marquez-Rodas I, McArthur GA, Ascierto PA, Long GV, Callahan MK, Postow MA, Grossmann K, Sznol M, Dreno B, Bastholt L, Yang A, Rollin LM, Horak C, Hodi FS, Wolchok JD (2015) Combined Nivolumab and Ipilimumab or monotherapy in untreated melanoma. N Engl J Med 373:23-34

3. Postow MA, Chesney J, Pavlick AC, Robert C, Grossmann K, McDermott D, Linette GP, Meyer N, Giguere JK, Agarwala SS, Shaheen M, Ernstoff MS, Minor D, Salama AK, Taylor M, Ott PA, Rollin LM, Horak C, Gagnier P, Wolchok JD, Hodi FS (2015) Nivolumab and ipilimumab versus ipilimumab in untreated melanoma. N Engl J Med 372:2006-2017

4. Ribas A, Puzanov I, Dummer R, Schadendorf D, Hamid O, Robert C, Hodi FS, Schachter J, Pavlick AC, Lewis KD, Cranmer LD, Blank CU, O’Day SJ, Ascierto PA, Salama AK, Margolin KA, Loquai C, Eigentler TK, Gangadhar TC, Carlino MS, Agarwala SS, Moschos SJ, Sosman JA, Goldinger SM, Shapira-Frommer R, Gonzalez R, Kirkwood JM, Wolchok JD, Eggermont A, Li XN, Zhou W, Zernhelt AM, Lis J, Ebbinghaus S, Kang SP, Daud A (2015) Pembrolizumab versus investigator-choice chemotherapy for ipilimumab-refractory melanoma (KEYNOTE-002): a randomised, controlled, phase 2 trial. Lancet Oncol 16:908-918

5. Robert C, Thomas L, Bondarenko I, O'Day S, Weber J, Garbe C, Lebbe C, Baurain JF, Testori A, Grob JJ, Davidson N, Richards J, Maio M, Hauschild A, Miller WH Jr., Gascon P, Lotem M, Harmankaya K, Ibrahim R, Francis S, Chen TT, Humphrey R, Hoos A, Wolchok JD (2011) Ipilimumab plus dacarbazine for previously untreated metastatic melanoma. N Engl J Med 364:2517-2526

6. Robert C, Long GV, Brady B, Dutriaux C, Maio M, Mortier L, Hassel JC, Rutkowski P, McNeil C, Kalinka-Warzocha E, Savage KJ, Hernberg MM, Lebbe C, Charles J, Mihalcioiu C, Chiarion-Sileni V, Mauch C, Cognetti F, Arance A, Schmidt H, Schadendorf D, Gogas H, Lundgren-Eriksson L, Horak C, Sharkey B, Waxman IM, Atkinson V, Ascierto PA (2015) Nivolumab in previously untreated melanoma without BRAF mutation. N Engl J Med 372:320-330

7. Weber JS, D'Angelo SP, Minor D, Hodi FS, Gutzmer R, Neyns B, Hoeller C, Khushalani NI, Miller WH Jr., Lao CD, Linette GP, Thomas L, Lorigan P, Grossmann KF, Hassel JC, Maio M, Sznol M, Ascierto PA, Mohr P, Chmielowski B, Bryce A, Svane IM, Grob JJ, Krackhardt AM, Horak C, Lambert A, Yang AS, Larkin J (2015) Nivolumab versus chemotherapy in patients with advanced melanoma who progressed after anti-CTLA-4 treatment
(CheckMate 037): a randomised, controlled, open-label, phase 3 trial. Lancet Oncol 16:375-384

8. Shaverdian N, Lisberg AE, Bornazyan K, Veruttipong D, Goldman JW, Formenti SC, Garon EB, Lee P (2017) Previous radiotherapy and the clinical activity and toxicity of pembrolizumab in the treatment of non-small-cell lung cancer: a secondary analysis of the KEYNOTE-001 phase 1 trial. Lancet Oncol 18:895-903

9. Hwang WL, Niemierko A, Hwang KL, Hubbeling H, Schapira E, Gainor JF, Keane FK (2018) Clinical outcomes in patients with metastatic lung cancer treated with PD-1/PD-L1 inhibitors and thoracic radiotherapy. JAMA Oncol 4:253-255

10. Rizvi NA, Hellmann MD, Snyder A, Kvistborg P, Makarov V, Havel JJ, Lee W, Yuan J, Wong P, Ho TS, Miller ML, Rekhtman N, Moreira AL, Ibrahim F, Bruggeman C, Gasmi B, Zappasodi R, Maeda Y, Sander C, Garon EB, Merghoub T, Wolchok JD, Schumacher TN, Chan TA (2015) Cancer immunology. Mutational landscape determines sensitivity to PD-1 blockade in non-small cell lung cancer. Science 348:124-128

11. Le DT, Durham JN, Smith KN, Wang H, Bartlett BR, Aulakh LK, Lu S, Kemberling H, Wilt C, Luber BS, Wong F, Azad NS, Rucki AA, Laheru D, Donehower R, Zaheer A, Fisher GA, Crocenzi TS, Lee JJ, Greten TF, Duffy AG, Ciombor KK, Eyring AD, Lam BH, Joe A, Kang SP, Holdhoff M, Danilova L, Cope L, Meyer C, Zhou S, Goldberg RM, Armstrong DK, Bever KM, Fader AN, Taube J, Housseau F, Spetzler D, Xiao N, Pardoll DM, Papadopoulos N, Kinzler KW, Eshleman JR, Vogelstein B, Anders RA, Diaz LA Jr. (2017) Mismatch repair deficiency predicts response of solid tumors to PD-1 blockade. Science 357:409-413

12. Alexandrov LB, Nik-Zainal S, Wedge DC, Aparicio SA, Behjati S, Biankin AV, Bignell GR, Bolli N, Borg A, Borresen-Dale AL, Boyault S, Burkhardt B, Butler AP, Caldas C, Davies HR, Desmedt C, Eils R, Eyfjord JE, Foekens JA, Greaves M, Hosoda F, Hutter B, Ilicic T, Imbeaud S, Imielinski M, Jager N, Jones DT, Jones D, Knappskog S, Kool M, Lakhani SR, Lopez-Otin C, Martin S, Munshi NC, Nakamura H, Northcott PA, Pajic M, Papaemmanuil E, Paradiso A, Pearson JV, Puente XS, Raine K, Ramakrishna M, Richardson AL, Richter J, Rosenstiel P, Schlesner M, Schumacher TN, Span PN, Teague JW, Totoki Y, Tutt AN, Valdes-Mas R, van Buuren MM, van 't Veer L, Vincent-Salomon A, Waddell N, Yates LR, Australian Pancreatic Cancer Genome, ICGC Breast Cancer Consortium, ICGC MMML-Seq Consortium, ICGC PedBrain, ZucmanRossi J, Futreal PA, McDermott U, Lichter P, Meyerson M, Grimmond SM, Siebert R, Campo E, Shibata T, Pfister SM, Campbell PJ, Stratton MR (2013) Signatures of mutational processes in human cancer. Nature 500:415-421

13. Lawrence MS, Stojanov P, Polak P, Kryukov GV, Cibulskis K, Sivachenko A, Carter SL, Stewart C, Mermel CH, Roberts SA, Kiezun A, Hammerman PS, McKenna A, Drier Y, Zou L, Ramos AH, Pugh TJ, Stransky N, Helman E, Kim J, Sougnez C, Ambrogio L, Nickerson E, Shefler E, Cortes ML, Auclair D, Saksena G, Voet D, Noble M, DiCara D, Lin P, Lichtenstein L, Heiman DI, Fennell T, Imielinski M, Hernandez B, Hodis E, Baca S, Dulak AM, Lohr J, Landau DA, Wu CJ, Melendez-Zajgla J, Hidalgo-Miranda A, Koren A, McCarroll SA, Mora J, Crompton B, Onofrio R, Parkin M, Winckler W, Ardlie K, Gabriel SB, Roberts CWM, Biegel JA, Stegmaier K, Bass AJ, Garraway LA, Meyerson M, Golub TR, Gordenin DA, Sunyaev S, Lander ES, Getz G (2013) Mutational heterogeneity in cancer and the search for new cancer-associated genes. Nature 499:214-218

14. Hellmann MD, Ciuleanu TE, Pluzanski A, Lee JS, Otterson GA, Audigier-Valette C, Minenza E, Linardou H, Burgers S, Salman P, Borghaei H, Ramalingam SS, Brahmer J, Reck M, O’Byrne KJ, Geese WJ, Green G, Chang H, Szustakowski J, Bhagavatheeswaran P, Healey D, Fu Y, Nathan F, Paz-Ares L (2018) Nivolumab plus Ipilimumab in lung cancer with a high tumor mutational burden. N Engl J Med 378:2093-2104 
15. Hall EJ, Giaccia AJ (2011) Radiobiology for the radiologist. Lippincott Williams \& Wilkins, Philadelphia, PA

16. Behjati S, Gundem G, Wedge DC, Roberts ND, Tarpey PS, Cooke SL, Van Loo P, Alexandrov LB, Ramakrishna M, Davies H, NikZainal S, Hardy C, Latimer C, Raine KM, Stebbings L, Menzies A, Jones D, Shepherd R, Butler AP, Teague JW, Jorgensen M, Khatri B, Pillay N, Shlien A, Futreal PA, Badie C, ICGC Prostate Group, McDermott U, Bova GS, Richardson AL, Flanagan AM, Stratton MR, Campbell PJ (2016) Mutational signatures of ionizing radiation in second malignancies. Nat Commun 7:12605

17. Herskind C, Ma L, Liu Q, Zhang B, Schneider F, Veldwijk MR, Wenz F (2017) Biology of high single doses of IORT: RBE, 5R's, and other biological aspects. Radiat Oncol 12:24

18. Knisely JP, Yu JB, Flanigan J, Sznol M, Kluger HM, Chiang VL (2012) Radiosurgery for melanoma brain metastases in the ipilimumab era and the possibility of longer survival. J Neurosurg 117:227-233

19. Silk AW, Bassetti MF, West BT, Tsien CI, Lao CD (2013) Ipilimumab and radiation therapy for melanoma brain metastases. Cancer Med 2:899-906

20. Tazi K, Hathaway A, Chiuzan C, Shirai K (2015) Survival of melanoma patients with brain metastases treated with ipilimumab and stereotactic radiosurgery. Cancer Med 4:1-6

21. Theelen W, Peulen H, Lalezari F, de Vries J, De Langen J, Aerts J, Monkhorst K, Baas P (2018) Randomized phase II study of pembrolizumab after stereotactic body radiotherapy (SBRT) versus pembrolizumab alone in patients with advanced non-small cell lung cancer: the PEMBRO-RT study. J Clin Oncol 36(suppl; abstr 9023)

22. Xie G, Gu D, Zhang L, Chen S, Wu D (2017) A rapid and systemic complete response to stereotactic body radiation therapy and pembrolizumab in a patient with metastatic renal cell carcinoma. Cancer Biol Ther 18:547-551
23. Twyman-Saint Victor C, Rech AJ, Maity A, Rengan R, Pauken KE, Stelekati E, Benci JL, Xu B, Dada H, Odorizzi PM, Herati RS, Mansfield KD, Patsch D, Amaravadi RK, Schuchter LM, Ishwaran H, Mick R, Pryma DA, Xu X, Feldman MD, Gangadhar TC, Hahn SM, Wherry EJ, Vonderheide RH, Minn AJ (2015) Radiation and dual checkpoint blockade activate non-redundant immune mechanisms in cancer. Nature 520:373-377

24. Herskind C, Wenz F, Giordano FA (2017) Immunotherapy combined with large fractions of radiotherapy: stereotactic radiosurgery for brain metastases-implications for Intraoperative radiotherapy after resection. Front Oncol 7:147

25. Demaria S, Formenti SC (2012) Radiation as an immunological adjuvant: current evidence on dose and fractionation. Front Oncol $2: 153$

26. Ruckert M, Deloch L, Fietkau R, Frey B, Hecht M, Gaipl US (2018) Immune modulatory effects of radiotherapy as basis for well-reasoned radioimmunotherapies. Strahlenther Onkol 194:509-519

27. Frey B, Ruckert M, Deloch L, Ruhle PF, Derer A, Fietkau R, Gaipl US (2017) Immunomodulation by ionizing radiation-impact for design of radio-immunotherapies and for treatment of inflammatory diseases. Immunol Rev 280:231-248

28. Frey B, Rubner Y, Wunderlich R, Weiss EM, Pockley AG, Fietkau R, Gaipl US (2012) Induction of abscopal anti-tumor immunity and immunogenic tumor cell death by ionizing irradiation-implications for cancer therapies. Curr Med Chem 19:1751-1764

29. Galluzzi L, Buque A, Kepp O, Zitvogel L, Kroemer G (2017) Reply: immunosuppressive cell death in cancer. Nat Rev Immunol 17:402

30. Blank CU, Haanen JB, Ribas A, Schumacher TN (2016) The "cancer immunogram". Science 352:658-660 